Yosuke Harada • Kouichi Ozaki • Mikio Suzuki

Tsutomu Fujiwara • Ei-ichi Takahashi

Yusuke Nakamura $\cdot$ Akira Tanigami

\title{
Complete cDNA sequence and genomic organization of a human pancreas- specific gene homologous to Caenorhabditis elegans sel-1
}

Received: March 29, 1999 / Accepted: May 11, 1999

\begin{abstract}
We have isolated the complete cDNA of a human SEL-1L gene, termed TSA305, that is abundantly expressed only in the pancreas. The cDNA contained an open reading frame of 2382 nucleotides, encoding a deduced protein of 794 amino acids whose predicted sequence showed $46 \%$ identity and $64 \%$ similarity with SEL-1 of Caenorhabditis elegans. SEL-1 is thought to be a negative regulator of the NOTCH, LIN-12, and GLP-1 receptors, which are required for differentiation and maturation of cells as well as cell-cell interactions during development in $C$. elegans. The degree of homology among these proteins suggests that the TSA305 gene product may be a member of the SEL-1 family and therefore involved in downregulation of mammalian Notch signaling. Direct sequencing revealed at least 20 coding exons in TSA305. We localized the gene to chromosome bands $14 \mathrm{q} 24.3-\mathrm{q} 31$ by radiation hybrid (RH) mapping and fluorescence in situ hybridization (FISH). The IDDM11 locus has been mapped in this region, and TSA305 may represent a candidate gene for predisposition in some families whose insulin-dependent diabetes is not linked to the HLA locus.
\end{abstract}

Key words Pancreas-specific gene $\cdot \mathrm{SEL}-1 \cdot \mathrm{SEL}-1 \mathrm{~L} \cdot$ Notch signaling $\cdot I D D M 11 \cdot 14 \mathrm{q} 24.3-\mathrm{q} 31$

\section{Introduction}

The sel-1 gene of Caenorhabditis elegans encodes a predicted extracellular protein identified as an extragenic sup-

Y. Harada $\cdot$ K. Ozaki $\cdot$ M. Suzuki $\cdot$ T. Fujiwara $\cdot$ E. Takahashi $\cdot$ A. Tanigami $(\square)$

Otsuka GEN Research Institute, Otsuka Pharmaceutical Co., Ltd., 463-10 Kagasuno, Kawauchi-cho, Tokushima 771-0192, Japan

Tel. +81-88-665-2888; Fax +81-88-637-1035

e-mail: atanigam@otsuka.gr.jp

Y. Nakamura

Laboratory of Molecular Medicine, Human Genome Center, Institute of Medical Science, University of Tokyo, Tokyo, Japan pressor of a lin-12 hypomorphic mutant (Grant and Greenwald 1996). The C. elegans lin-12 gene encodes a member of the LIN-12/NOTCH receptor family (Greenwald and Rubin 1992; Artavanis-Tsakonas et al. 1995). The members of this family include $C$. elegans GLP1 and Drosophila Notch. Two vertebrate homologs, murine int-3 and human $T A N-1$, have been implicated in some cancers (Ellisen et al. 1991; Jhappan et al. 1992; Robbins et al. 1992). Abnormalities in genes that are expressed only in certain tissue(s) might lead to organ-specific diseases. For example, because insulin-dependent diabetes mellitus (IDDM) is caused by the destruction of the insulin-producing $\beta$-cells in the islets of Langerhans in the pancreas, defects in genes that regulate pancreatic islet development and insulin biosynthesis probably contribute to the pathogenesis of this disorder.

Among gastrointestinal malignancies, pancreatic cancers have the poorest prognoses, being the fourth or fifth leading cause of cancer-related deaths in Japan and Western countries (Poston et al. 1991). Determining the genes that are involved in such tissue-specific disorders may ultimately allow development of useful diagnostic tools and more effective therapies. The differential display technique (DD) provides a rapid and efficient way to isolate genes that are differently expressed among various tissues. We have isolated several human pancreas-specific genes in this way, and demonstrated that DD gives access to transcripts as rare as 1 in 1 million molecules of total mRNA (Ozaki et al. 1996, 1998). In the present study, a continuation of that effort, we identified a pancreas-specific transcript and subsequently cloned its full-length cDNA, which encoded a protein similar to SEL-1 of $C$. elegans. We mapped the pancreas-specific gene to the distal portion (q24.3-q31) of the long arm of human chromosome 14 , a region containing one (IDDM11) of several loci associated with insulin-dependent diabetes mellitus. Toward exploring the regulatory role of this gene and identifying mutations that might affect its function with respect to diabetes and cancer, we determined all exon-intron junctions and analyzed its pattern of expression in normal human tissues. 


\section{Materials and methods}

\section{Differential display}

The differential display (DD) procedure was carried out essentially as described by Liang and Pardee (1992). Because the polymerase chain reaction (PCR) products contained plural bands of the same size, the reamplified products were electrophoresed on agarose gels containing a bisbenzimide-PEG (polyethylene glycol) conjugate, H.A.-Yellow (Hanse Analytik GmbH, Bremen, Germany); as this reagent specifically adheres to adenine/ thymine (A/T) bases, target genes can be separated from background bands. The desired cDNA fragments were subcloned in the manner described previously (Ozaki et al. 1996). Nucleotide sequences were determined with an ABI 377 auto-sequencer (Applied Biosystems, Foster City, CA, USA).

\section{Northern blot analysis}

Human multiple-tissue Northern (MTN) blots I and II (Clontech, Palo Alto, CA, USA) were prehybridized and then hybridized with an $\alpha-\left[{ }^{32} \mathrm{P}\right] \mathrm{dCTP}$-labeled fragment (nucleotides 1-2382) of a cDNA (provisionally designated TSA305) that had been isolated through DD. We used the manufacturer's protocol with a random-labeling kit (BcaBEST Labeling Kit; Takara, Kyoto, Japan). Washed membranes were autoradiographed for $12 \mathrm{~h}$ at $-80^{\circ} \mathrm{C}$.

\section{Screening of cDNA and sequencing}

A human pancreatic cDNA library was constructed using oligo $\left(\mathrm{dT}_{16}\right)+$ random hexamer-primed pancreatic cDNA and Uni-ZAPXR (Stratagene, La Jolla, CA, USA). A total of $1 \times 10^{6}$ clones were screened with an $\alpha-\left[{ }^{32} \mathrm{P}\right] \mathrm{dCTP}$-labeled fragment (nucleotides 7514-7885) of TSA305 cDNA. Positive clones were selected and their insert DNAs were excised in vivo in pBluescript II SK(-) (Stratagene) according to the supplier's recommendation. Sequencing was performed with an ABI 377 auto-sequencer.

\section{Radiation hybrid mapping}

Using primer 1 (5'-GGAGGTACTGCTGTGTAATG-3') and primer $2\left(5^{\prime}\right.$-TCCACTCAACTTACATGAG-3') generated from the $3^{\prime}$-noncoding region of TSA305 cDNA, we analyzed a panel of 93 radiation hybrid $(\mathrm{RH})$ clones (GeneBridge 4; Research Genetics, Huntsville, AL, USA) by PCR. Amplified products were separated according to size by electrophoresis on $1.5 \%$ agarose gels. Each hybrid clone was scored as positive or negative according to the presence or absence, respectively, of a 282-bp band. The data from the panel were analyzed by two-point maximum-likelihood analysis software, RHMAPPER, through the URL at http:// www-genome.wi.mit.edu/cgi-bin/contig/rhmapper.pl.
Cosmid, BAC screening, and genomic organization

To prepare a probe for fluorescence in situ hybridization (FISH) analysis, we isolated a cosmid (0202G02) containing sequences corresponding to the TSA305 gene by screening a total of 153,600 cosmid clones by PCR (Watanabe et al. 1996), using primers 1 and 2 (above). Cycling conditions were $94^{\circ} \mathrm{C}$ for $2 \mathrm{~min}$, followed by 35 cycles of denaturing at $94^{\circ} \mathrm{C}$ for $30 \mathrm{~s}$, annealing at $58^{\circ} \mathrm{C}$ for $30 \mathrm{~s}$, and extension at $72^{\circ} \mathrm{C}$ for $40 \mathrm{~s}$. To determine genomic organization, a clone containing sequences corresponding to the $5^{\prime}$-portion of TSA305 cDNA was isolated from the human BAC library (Research Genetics) by PCR screening using primer 3 (5'-GCGAAGGCGACAGCTCTA-3') and primer 4 (5'-CCGAGGACGCCGAGGCCAA-3') for 35 cycles of $94^{\circ} \mathrm{C}$ for $30 \mathrm{~s}, 58^{\circ} \mathrm{C}$ for $30 \mathrm{~s}$, and $72^{\circ} \mathrm{C}$ for $40 \mathrm{~s}$. The exon-intron boundaries were defined by directly sequencing the BAC (184M20) and cosmid DNAs using appropriate primers for each exon. For the region not covered by the BAC and cosmid clones, we defined exon-intron junctions by sequencing PCR products that had been amplified from human genomic DNA using exonic primers flanking the introns.

\section{Results}

\section{Differential display}

To identify novel tissue-specific genes, we compared display patterns using mRNAs that had been isolated from human brain, lung, liver, pancreas, stomach, prostate, spleen, heart, kidney, thymus, placenta, testis, skeletal muscle, and lymph node. One primer combination (arbitrary primer $5^{\prime}$ CTTGATTGCC-3' and 3'-anchored oligo-dT primer 5'$\left.\mathrm{GT}_{15}(\mathrm{~A} / \mathrm{G} / \mathrm{C}) \mathrm{A}-3^{\prime}\right)$ among the 116 primer sets we examined identified a pancreas-specific DNA fragment of TSA305 (Fig. 1A).

\section{Northern blot analysis with cloned fragments}

To confirm the pattern observed in the differential display, we examined expression of TSA305 in various adult human tissues. Northern blots revealed a single transcript of about $7.8 \mathrm{~kb}$, specifically in pancreas. Although additional transcripts of $4.0,3.5,1.5$, and $0.8 \mathrm{~kb}$ were detected in pancreas, only the $7.8-\mathrm{kb}$ transcript appeared in other tissues, faintly but ubiquitously, after long exposure of the X-ray film (Fig. 1B).

\section{Cloning and sequencing of full-length cDNA}

By screening a human pancreas cDNA library $\left(1 \times 10^{6} \mathrm{pfu}\right)$ using TSA305 as the probe, we identified about 100 positive clones. On the basis of this result, we estimated the abundance of transcript among total pancreatic mRNAs to be 
$0.01 \%$. After checking the sizes of inserts in 20 candidate clones, the clone that carried the largest cDNA insert (about $3 \mathrm{~kb}$ ) was sequenced. To amplify the missing $5^{\prime}$ portion, we performed $5^{\prime}$-RACE experiments (5'-rapid amplification of cDNA ends (Frohman et al. 1988) several times with the 5'-AmpliFinder kit (Clontech). The assembled cDNA sequence contained 7885 nucleotides (GenBank accession number, AB020335), which included 2382 nucleotides of open reading frame encoding a protein of 794 amino acids with a calculated molecular weight of $88,750 \mathrm{Da}$. Using the BLAST program to search public databases, we found $97 \%$ identity to the sequence of the "SEL-1L" gene previously reported by Biunno et al. (1997) under accession number U11037; the major difference was that our sequence contained a long $5^{\prime}$-portion upstream. The BLAST search also revealed significant similarity of our predicted amino acid sequence to SEL-1, the product of



B
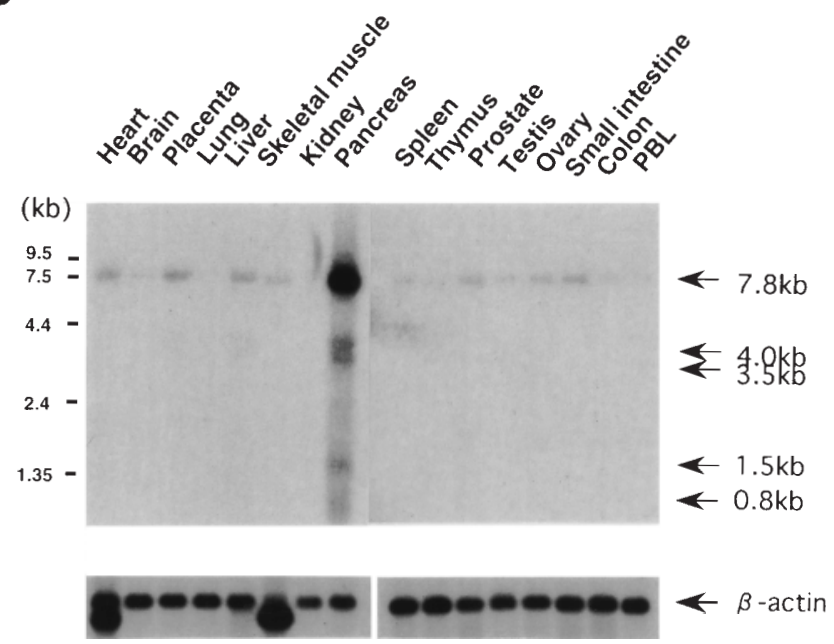

Fig. 1. A Differential display. The arrow indicates a pancreas-specific band (TSA305) among 14 normal human tissues. B Northern blotting of TSA305. Size markers are shown on the left, and the sizes of TSA305 transcripts are indicated at right. Beta-actin expression was measured as control. PBL, peripheral blood lymphocytes the sel-1 gene of C. elegans (46\% identity and $64 \%$ similarity; Fig. 2). Furthermore, this gene is highly homologous (92\% identity; Fig. 2 ) to the deduced amino acid sequence of murine SEL-1L (mSEL-1L) reported by Donoviel et al. (1998). The PSORT program (Horton and Nakai 1996) revealed a hydrophobic signal sequence near the N-terminus of the deduced amino acid sequence, and two potential transmembrane domains near the C-terminus (Fig. 3).

\section{Chromosomal localization of TSA305}

We localized TSA305 by radiation hybrid (RH) mapping (Walter et al. 1994), using TSA305-specific primers to analyze a panel of $93 \mathrm{RH}$ clones by PCR to generate the following data: 0011000100000000100000001000000001101100 0110100000000200000100000200000000010121000 . We determined that the TSA305 gene was most tightly linked to D14S76, a marker located at 14q24.3. We also performed FISH using cosmid clone $0202 \mathrm{G} 02$ as a probe. We examined 100 typical R-banded metaphase spreads, and observed doublet signals only at the q24.3-q31 bands of chromosome 14 (Fig. 4).

\section{Genomic organization}

We used BAC and cosmid clones, and PCR products amplified from human genomic DNA (see Materials and methods), to characterize the exon-intron boundaries of the TSA305 gene (Table 1). The sequences of all splice sites conformed to the GT/AG rule (Breathnach and Chambon 1981). Our genomic analysis revealed that the gene contained at least 20 exons. Accession numbers of the TSA305 sequence are AB024747, AB024748, $\mathrm{AB} 024749, \mathrm{AB} 024750$, AB024751, AB024752, AB024753, $\mathrm{AB} 024754, \mathrm{AB} 024755$, AB024756, AB024757, AB024758, $\mathrm{AB} 024759, \mathrm{AB} 024760, \mathrm{AB} 024761, \mathrm{AB} 024762$, and AB024763.

\section{Discussion}

We have described here the isolation and characterization of a human complete $S E L-1 L$ gene (TSA305), which is expressed most strongly in the pancreas. While this manuscript was being prepared, Biunno et al. (1997) reported the partial sequence of a human cDNA corresponding to $S E L$ 1 , its chromosomal localization, and its pattern of expression in pancreatic carcinomas. The primary sequence of their predicted product (SEL-1L) corresponded to the codons represented by amino acids $640-699$ in Fig. 3. The expression profile and our RH and FISH mapping (14q24.3-q31) of the TSA305 gene were concordant with previous results reported by the Biunno group. However, the amino acid sequence of SEL-1L seemed to lack about two-thirds of the N-terminal (amino acids 1-639) of TSA305 and part of the C-terminal region (amino acids 
700-794).

The predicted gene product bears significant similarity to the $C$. elegans SEL-1 protein, which was identified as an extragenic suppressor of hypomorphic lin-12 and glp-1 mutants (Barth and Iva 1996). The lin-12 gene of C. elegans encodes a member of the LIN-12/NOTCH family of putative receptor proteins; lin-12 mutations affect binary cellfate decisions in many different cell types and at many different developmental stages of the worm (Greenwald et al. 1983). SEL-1 may interact with LIN-12 and GLP-1 or their ligands to suppress the function of those proteins. Mammalian members of the LIN-12/NOTCH family include TAN-1, the human homolog of the Drosophila Notch, and murine INT-3. TAN-1 is often broken by chromosomal translocations in T-lymphoblastic neoplasms, and INT-3 transforms mammary epithelial cells in the mouse (Ellisen et al. 1991; Robbins et al. 1992). Although the function of the TSA305 gene product is unknown, its similarity to SEL1 , which is capable only of modulating LIN-12 and GLP-1 activities, indicates that the human protein may play a significant role in development or progression of pancreatic carcinogenesis or normal pancreatic development.

The deduced TSA305 protein contained a potential hydrophobic sequence near the $\mathrm{N}$-terminus and two transmembrane domains near the C-terminus (see Fig. 3). Hydrophobic amino-terminal regions are thought to be secretory signals (von Heijne 1986). However, the protein may be anchored at the plasma membrane by its C-terminal hydrophobic sequences. Five N-glycosylation sites (NX[S/ $\mathrm{T}]$ ) were scattered in the predicted extracellular region (amino acids 1-700). A type 2 fibronectin collagen-binding domain was found at position 127-168 in the predicted primary sequence of the TSA305 gene product. This domain exhibits substantial homology to metalloproteinase-9 (MP9) and matrix metalloproteinase-2 (MMP-2) of rabbit osteoclasts (Tezuka et al. 1994), to gelatinase of chicken embryo fibroblasts (Aimes et al. 1994), and to murine type IV collagenase (Reponen et al. 1992) (see Fig. 5). The sequence of this domain is conserved between human TSA305 and its murine homolog, but not in SEL-1 (see Fig. 2).

Proteolytic degradation of various constituents of the extracellular matrix and basement membranes plays an important role in tissue-restructuring processes such as cell migration, morphogenesis, wound healing, angiogenesis, and tumor invasion (Liotta et al. 1991). Type IV collagen is the major structural component of basement membranes (Martin et al. 1988). Therefore the collagenolytic activity of type IV collagenases such as MMP-9 and gelatinase B is viewed as a critical component of the metastatic process. However, as TSA305 does not retain the catalytic domain present in type IV collagenases, its collagen-binding domain may function simply through binding to the surrounding extracellular matrix.

According to the results of our Northern blot analysis, a 7.8-kb transcript of TSA305 was present predominantly in pancreas, although it appeared in all human tissues examined after long exposure of the X-ray film. This result suggests that the principal role of TSA305 may be specific to the pancreas, especially because several smaller transcripts $(4.0,3.5,1.5$, and $0.8 \mathrm{~kb})$ were detected only in pancreatic mRNA. When we used a probe constituting approximately $3 \mathrm{~kb}$ of the $3^{\prime}$-region, Northern analysis revealed only a single 7.8-kb transcript (data not shown). Therefore the smaller transcripts may be created by alternative splicing in the $3^{\prime}$-nontranslated regions, or they may be derived from unknown genes that are highly homologous to TSA305.

Our mapping experiments localized TSA305 to chromosome bands 14q24.3-q31, where variant allele(s) responsible for one type of insulin-dependent diabetes mellitus (IDDM11) have been mapped. Significant linkage heterogeneity between HLA-defined subsets of families has suggested that IDDM11 may be an important susceptibility

Table 1. Exon/intron junction sequences of the TSA305 gene

\begin{tabular}{lllr}
\hline Exon & Splice acceptor & Splice donor & Exon length (bp) \\
\hline 1 & & GCGTCCTCGG/gtcagtatcc & $>70$ \\
2 & tcctttgcag/ATGAAGAAGG & AGATTCCAAG/gtatgtacta & 38 \\
3 & atattctcag/ACTACTTTGA & CGGAAACCAG/gtagtctgga & 232 \\
4 & gtcattccag/CTTTGACCGC & TTTTGTGAAA/gtaagtattg & 168 \\
5 & ttgttttag/CTGAAGAAGA & AAAAAAGAGA/gtaggtagca & 106 \\
6 & acatttgaag/AGCATATCGG & GGGACAGACT/gtaagtacat & 163 \\
7 & tctattcag/GCTCTTGGCT & TCAGGCAAAG/gtaatactat & 54 \\
8 & tctttcacag/GCTCTTGTAT & CATGGTTTG/gtaagtagac & 60 \\
9 & tgtttacag/GGTTACAGAT & GCCAATCATG/gtatctatgt & 83 \\
10 & tctattcag/TTGCTAGTGA & ACAAGCACAG/gtacgtgttt & 155 \\
11 & tcatttcag/GTTGGTCTTG & GAATCATCAG/gtaactaccc & 57 \\
12 & tttctcatag/AGAGCATTTG & TTTGGGAAAG/gtactgtaca & 69 \\
13 & tgttttcag/ATGTATTCGG & TGCTGACATG/gtaaggcttt & 78 \\
14 & tgctttgcag/GGCAACCCAG & ATGTACTATA/gtaagtaaca & 151 \\
15 & tttcttacag/ATGGCATTGG & TGCAGTGGAG/gtaaggtctt & 149 \\
16 & cctcacacag/TTGTTTAAGA & CTTGATCAGAgtaaggttca & 166 \\
17 & tttctgtag/GAGAAGCAAG & GCCTCTCAAG/gtaatgataa & 75 \\
18 & tttgttaag/GCTATACTGT & CATTAAACAG/gtgagtgtgg & 173 \\
19 & tgtattatag/GATATTCACC & GGAAACAAAC/gtaagtggtc & 129 \\
20 & tttcattaag/ATTCGAGATA & & $>207$ \\
\hline
\end{tabular}


hTSA305 mSEL-1L

SEL-1

hTSA305 MSEL-1L

SEL-1

hTSA305 mSEL-1L SEL-1

hTSA305 mSEL-1L

SEL-1

hTSA305 MSEL-1L

SEL-1

hTSA305 MSEL-1L 234 SEL-1

hTSA305 mSEL-1L SEL-1

hTSA305 MSEL-1L SEL-1

hTSA305 MSEL-1L SEL-1

hTSA305 mSEL-1L SEL-1

hTSA305 mSEL-1L SEL-1

hTSA305 mSEL-1L SEL-1

hTSA305 565 mSEL-1L 561 SEL-1

hTSA305 610 mSEL-1L 606 SEL-1

hTSA305 658 mSEL-1L 654 SEL-1

hTSA305 706 MSEL-1L 702 SEL-1 629

hTSA305 754 MSEL-1L 750 SEL-1 37 72

MR VR I GL T L L L C A V L LS LASAS D DE E G QDE S LDS KT T L T S DESVKD

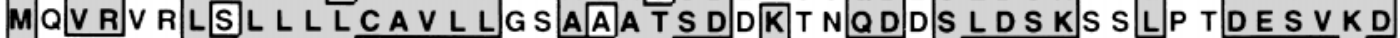



H T TAGR VVAGQ I FL DSEESELESS I QEEEDSLKSQEGES V TED ISFLE H T T T GKVVAGQ I FVDSEEAEVESL LQDEE DSSIKTQE - . . . E E I S F LE

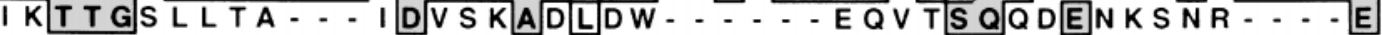

S P N PEN ND YEEP KK VRKPALTA IEGTAHGEPCHFPFLFLDKEYDECTS S P N P S S K T Y E E L R VRKPVLTA I EGTAHGEPCHFPFLFLDKEYDECTS

DGRE DGR LWCA T TYDYKA DEKWG FCETEEE AAKRRQMQEAEMMYQTM DGREDGRLWCAT TYDYK T DEKWGFCETEE DAKRRQMQEAEM I Y Q A GM

K I LN - - GSNK KS QKREAYRYLQKAASMNHTKALERVSYALLFGDYLPQ K I LN - - GS NRKS QKREAYRYLQKAAGMNHTKALERVSYALLFGDYLTQ AY IERGKGHGREGRVAAHRV FERAAA QGHQEARKAVAFS QMEGDYSRW

N I QAAREM FEKL TEEGSPKGQTALGFLYASGLGVN-SSQAKALVYYTF N I QAAKEM FEKLTEEGSPKGQTGLG L Y ASGLGVN - S SQAKALVYYTF SI Q EAKT VLF DLLEK NGS P D Q LA L G FMHGAG G VEKSNQAKALVYYMF

GALGGN L I AHMV LGYRYWAG I GVLQSCESALTHYRLVANHVASDISLT GA L G G L I A HM I LGYRYWAG I GV LQSCESALTHYRLVANHVASDISLT

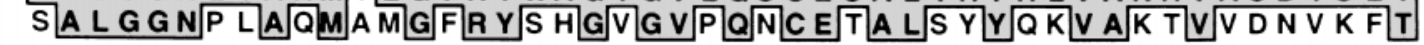

GGSVVQR IRLPDEVENPGMN- - - SGMLEE DL I QYYQFLAEKGDVQAQ 329 G GS V VQR IR LPDEVENPGMN - . - S GM LGGDL I QY Y QFLAEKGDVQAQ



377 373 293

25 341

473 469
389

517 513 37$$
\text { A }
$$$$
485
$$

6 533

$$
\text { V }
$$

$$
1
$$

VGLGQLHLHGGRGVEQNHQRAFDYFNLAANAGNSHAMAFLGKMYSEGS $V G L G Q L H L H G G R G V E Q N H Q R A F D Y F N L A A N A G N S H A M A F L G K M Y S E G S$ LG L G O I L L A GR GL NQN F L A FRY L LAA SE SSADAL TYLGKMYLDG T

D IVPQSNETALHYFKKAADMGNPVGQSGLGMAYLYGRGVQVNYDLALK DIVPQSNETALHYFKKAADMGNPVGQSGLGMAYLYGRGVQVNYDLALK

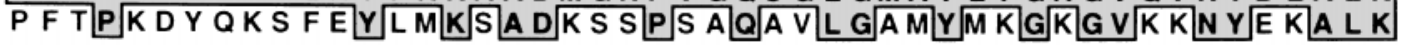

YFQKAAEQGWVDGQLQLGSMYYNG - . - - GVKRDYKQALKYFNLASQG YFQKAAEQGWVDGQLQLGSMYYNGI... GVKRDYKQALKYFNLASQG

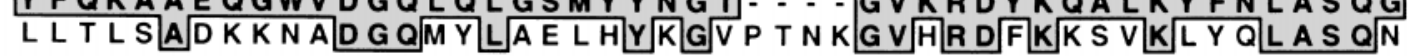

GH I LAFYNLAQMHAS GTGVMRSCHTAVELFKNVCERGRWSERLMTAYN GH I LAFHN LAQMHANGTGVMRS CQTGVELFKN V CE RSRWSERLMTAYN

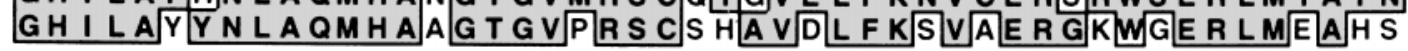

SYKDG DYNAAV I QYLLLAEQGYEVAQSNAAF ILDQREAS IV - - - GENE SYKDEDYNAAVVQYLLLAEQGYEVAQSNAAFILDQREAS I V. - - GENE

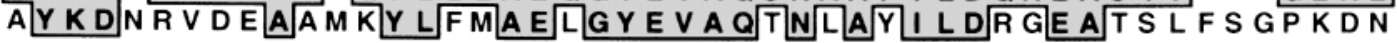

TYPRALLHWNRAASQGYTVAR IKLGDYHFYGFGTDVDYETAF I HYRLA TYPRALLHWNRAASQGYTVAR IKLGDYHFYGFGTDVDYETAF I HYRLA

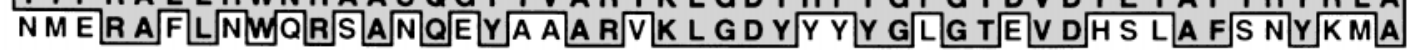

SEQQHSAQAMFNLGYMHEKGLG IKQDIHLAKRFYDMAAEASPDAQVPV SEQQHSAQAMFNLGYMHEKGLG IKQD I HLAKRFYDMAAEASPDAQVPV

FLALCKLGVVYFLQY IRETT IRDMFTQLDMDQLLGPEWDLYLMT I IAL

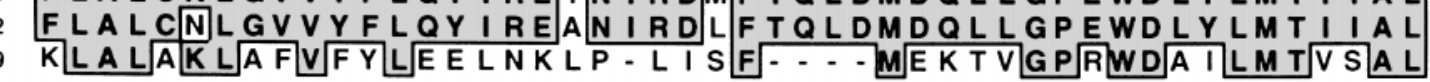

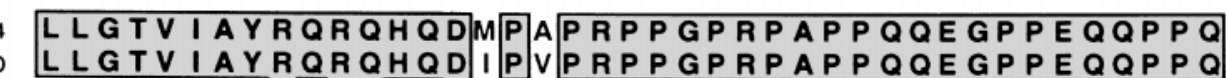
672 V - P LFLFWR HR Q N D $\ldots \ldots$

Fig. 2. Comparison of predicted amino acid sequences of human TSA305, murine SEL-1L, and Caenorhabditis elegans SEL-1. Identities are indicated by boxed letters 
1 ATGCGGTTCGGATAGGGCTGACGCTGCTGCTGTGTGCGGTGCTGCTGAGCTTGGCCTCGGCGTCCTCGGATGAAGAAGGCAGCCAGGATGAATCCTTAGATTCCAAGACTACTTTGACA

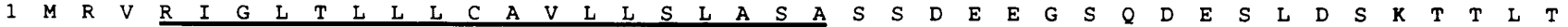

121 TCAGATGAGTCAGTAAAGGACCATACTACTGCAGGCAGAGTAGTTGCTGGTCAAATATTTCTTGATTCAGAAGAATCTGAATTAGAATCCTCTATTCAAGAAGAGGAAGACAGCCTCAAG

$\begin{array}{llllllllllllllllllllllllllllllllllllllllll}41 & S & D & E & S & V & K & D & H & T & T & A & G & R & V & V & A & G & Q & I & F & L & D & S & E & E & S & E & L & E & S & S & I & Q & E & E & E & D & S & L & K\end{array}$

241 AGCCAAGAGGGGAAAGTGTCACAGAAGATATCAGCTTTCTAGAGTCTCCAAATCCAGAAAACAAGGACTATGAAGAGCCAAAGAAAGTACGGAAACCAGCTTTGACCGCCATTGAAGGC

$\begin{array}{lllllllllllllllllllllllllllllllllllllllllll}81 & S & Q & E & G & E & S & V & T & E & D & I & S & F & L & E & S & P & N & P & E & N & K & D & Y & E & E & P & K & K & V & R & K & P & A & L & T & A & I & E & G\end{array}$

361 ACAGCACATGGGGAGCCCTGCCACTTCCCTTTTCTTTTCCTAGATAAGGAGTATGATGAATGTACATCAGATGGGAGGGAAGATGGCAGACTGTGGTGTGCTACAACCTATGACTACAAA

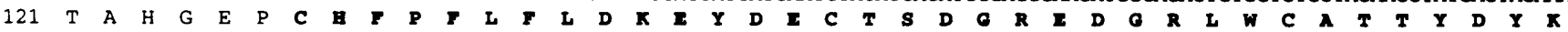

481 GCAGATGAAAAGTGGGCTTTTGTGAAACTGAAGAAGAGGCTGCTAAGAGACGGCAGATGCAGGAAGCAGAAATGATGTATCAAACTGGAATGAAAATCCTTAATGGAAGCAATAAGAAA



600 AGCCAAAAAAGAGAAGCATATCGGTATCTCCAAAAGGCAGCAAGCATGAACCATACCAAAGCCCTGGAGAGAGTGTCATATGCTCTTTTATTTGGTGATTACTTGCCACAGAATATCCAG

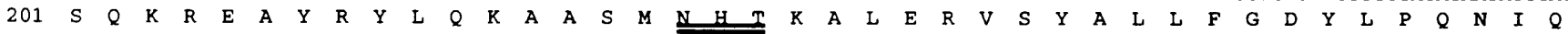

721 GCAGCGAGAGAGATGTTTGAGAAGCTGACTGAGGAAGGCTCTCCCAAGGGACAGACTGCTCTTGGCTTTCTGTATGCCTCTGGACTTGGTGTTAATTCAAGTCAGGCAAAGGCTCTTGTA


841 TATTATACATTTGGAGCTCTTGGGGGCAATCTAATAGCCCACATGGTTTTGGGTTACAGATACTGGGCTGGCATCGGCGTCCTCCAGAGTTGTGAATCTGCCCTGACTCACTATCGTCTT

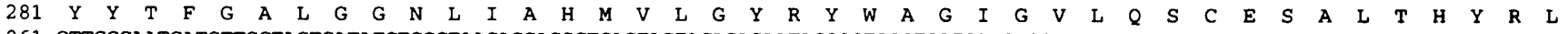
961 GTTGCCAATCATGTTGCTAGTGATATCTCGCTAACAGGAGGCTCAGTAGTACAGAGAATACGGCTGCCTGATGAAGTGGAAAATCCAGGAATGAACAGTGGAATGCTAGAAGAAGATTTG

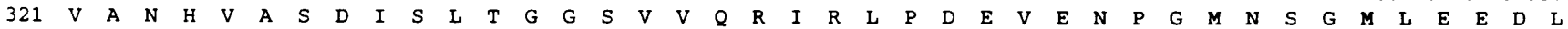

1081 ATTCAATATTACCAGTTCCTAGCTGAAAAAGGTGATGTACAAGCACAGGTTGGTCTTGGACAACTGCACCTGCACGGAGGGCGTGGAGTAGAACAGAATCATCAGAGAGCATTTGACTAC

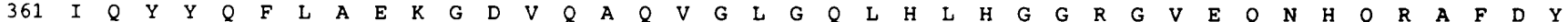
1201 TTCAATTTAGCAGCAAATGCTGGCAATTCACATGCCATGGCCTTTTTGGGAAAGATGTATTCGGAAGGAAGTGACATTGTACCTCAGAGTAATGAGACAGCTCTCCACTACTTTAAGAAA

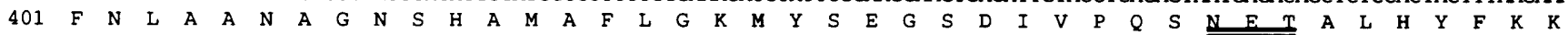
1321 GCTGCTGACATGGGCAACCCAGTTGGACAGAGTGGGCTTGGAATGGCCTACCTCTATGGGAGAGGAGTTCAAGTTAATTATGATCTAGCCCTTAAGTATTTCCAGAAAGCTGCTGAACAA $\begin{array}{llllllllllllllllllllllllllllllllllllllllllll}441 & A & A & D & M & G & N & P & V & G & Q & S & G & \text { L } & G & M & A & Y & \text { L } & Y & G & R & G & V & Q & V & N & Y & D & \text { L } & A & L & K & Y & F & Q & K & A & A & E & Q\end{array}$

1441 GGCTGGGTGGATGGGCAGCTACAGCTTGGTTCCATGTACTATAATGGCATTGGAGTCAAGAGAGATTATAAACAGGCCTTGAAGTATTTTAATTTAGCTTCTCAGGGAGGCCATATCTTG


1561 GCTTTCTATAACCTAGCTCAGATGCATGCCAGTGGCACCGGCGTGATGCGATCATGTCACACTGCAGTGGAGTTGTTTAAGAATGTATGTGAACGAGGCCGTTGGTCTGAAAGGCTTATG

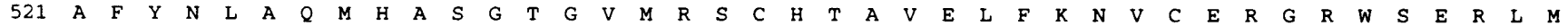
1681 ACTGCCTATAACAGCTATAAAGATGGCGATTACAATGCTGCAGTGATCCAGTACCTCCTCCTGGCTGAACAGGGCTATGAAGTGGCACAAAGCAATGCAGCCTTTATTCTTGATCAGAGA

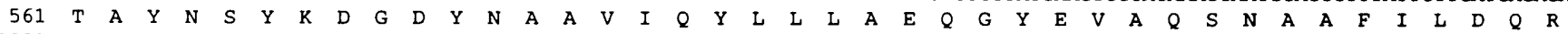
1801 GAAGCAAGCATTGTAGGTGAGAATGAAACTTATCCCAGAGCTTTGCTACATTGGAACAGGGCCGCCTCTCAAGGCTATACTGTGGCTAGAATTAAGCTCGGAGACTACCATTTCTATGGG

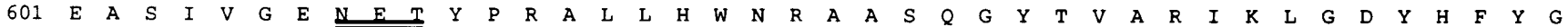
1921 TTTGGCACCGATGTAGATTATGAAACTGCATTTATTCATTACCGTCTGGCTTCTGAGCAGCAACACAGTGCACAAGCTATGTTTAATCTGGGATATATGCATGAGAAAGGACTGGGCATT

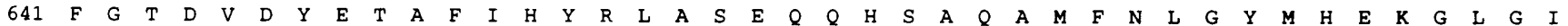
2041 AAACAGGATATTCACCTTGCGAAACGTTTTTATGACATGGCAGCTGAAGCCAGCCCAGATGCACAAGTTCCAGTCTTCCTAGCCCTCTGCAAATTGGGCGTCGTCTATTTCTTGCAGTAC

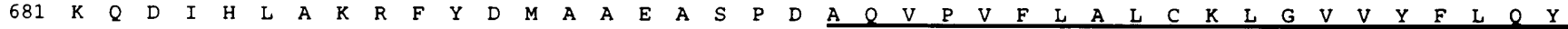
2161 ATACGGGAAACAAACATTCGAGATATGTTCACCCAACTTGATATGGACCAGCTTTTGGGACCTGAGTGGGACCTTTACCTCATGACCATCATTGCGCTGCTGTTGGGAACAGTCATAGCT

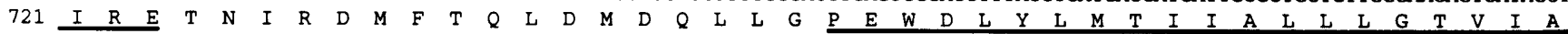
2281 TACAGGCAAAGGCAGCACCAAGACATGCCTGCACCCAGGCCTCCAGGGCCACGGCCAGCTCCACCCCAGCAGGAGGGCCACCAGAGCAGCAGCCACCACAGTAATAGGCACTGGGTCCA $\begin{array}{lllllllllllllllllllllllllllllllllllllllllll}761 & Y & R & Q & R & Q & H & Q & D & M & P & A & P & R & P & P & G & P & R & P & A & P & P & Q & Q & E & G & P & P & E & Q & Q & P & P & Q & *\end{array}$

Fig. 3. Nucleotide and amino acid sequences of TSA305. Numbering begins at the position of the translation-initiation codon; the termination codon (TAA) is indicated by an asterisk. A hydrophobic signal sequence at the N-terminus and transmembrane regions at the C-terminus are underlined, and potential N-glycosylation sites are double-underlined. Amino acids of the collagen-binding domain are indicated by bold type. The nucleotide sequence will appear in the DDBJ, GenBank, and EMBL databases with accession number AB020335

Fig. 4. Localization of TSA305 on metaphase chromosomes by FISH. The arrow indicates twin signals at $14 \mathrm{q} 24.3-\mathrm{q} 31$

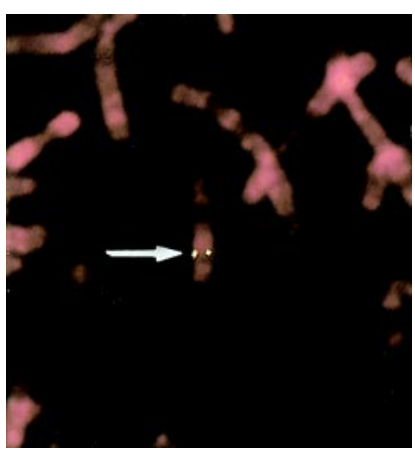

locus in diabetic families that lack a strong predisposition in the HLA region (Field et al. 1996). We are evaluating the intron-exon boundary sequences and pursuing functional analysis of TSA305 as steps toward discovering whether patients with IDDM or cancer carry mutations of the TSA305 gene.

Acknowledgments We thank all the staff members of the Otsuka GEN Research Institute for their technical assistance, gratefully acknowledging Fumiko Inoue for her excellent technical expertise; Atsushi
Kawai, Yuka Fujii, and Yoshiko Shimada for sequencing; Hiroichi Shinomiya for RH paneling; and Yasuo Irie for valuable suggestions. This work was supported in part by grants from the Ministry of Education, Science, Sports, and Culture of Japan (E.T.).

\section{References}

Aimes RT, French DL, Quigley JP (1994) Cloning of a 72 kDa matrix metalloproteinase (gelatinase) from chicken embryo fibroblasts using gene family PCR: expression of the gelatinase increases upon malignant transformation. Biochem J 300:729-736

Artavanis-Tsakonas S, Matsuno K, Fortini ME (1995) Notch signaling. Science 268:225-232

Barth G, Iva G (1996) The Caenorhabditis elegans sel-1 gene, a negative regulator of $l i n-12$ and $g l p-1$, encodes a predicted extracellular protein. Genetics 143:237-247

Biunno I, Appierto V, Cattaneo M, Leone BE, Balzano G, Socci C, Saccone S, Letizia A, Valle GD, Sgaramella V (1997) Isolation of a pancreas-specific gene located on human chromosome 14q31: expression analysis in human pancreatic ductal carcinomas. Genomics 46:284-286

Breathnach R, Chambon P (1981) Organization and expression of eukaryotic split genes coding for proteins. Annu Rev Biochem 50:349-383

Donoviel DB, Donoviel MS, Fan E, Hadjantonakis A, Bernstein A (1998) Cloning and characterization of Sel-11, a murine homolog of the C. elegans sel-1 gene. Mech Dev 78:203-207 


$\begin{array}{lll}\text { TSA305 } & 127 & \text { CHFPFLFLDKEYDECTSDGREDGR LWCATTYDYKADEKWGFC } \\ \text { h-COL4 } & 233 & \text { CKFPFLFNGKEYNSCTDTGRSDGFLWCSTTYNFEKDGKYGFC } \\ \text { m-COL4 } & 230 & \text { CHFPFTFEGR SYSACTTDGRNDGTPWCSTTADYDKDGKFGFC } \\ \text { GEL } & 98 & \text { CHFPFTFEGR SYSACTTDGRNDGTPWCSTTADYDKDGKFGF C } \\ \text { MP-9 } & 51 & \text { CVFPFVFLGKEYSTCTSDGRRDGRLWCATTSNFDTDKKWGFC } \\ \text { MMP-2 } & 233 & \text { CKFPFLFNGKEYTSCTDTGRSDGFLWCSTTYNFEKDGKYGFC }\end{array}$

Fig. 5. The predicted amino acid sequence of the collagen-binding domain of human TSA305, aligned with the sequences of human type IV collagenase (h-COL4), a murine type IV collagenase (m-COL4),

Ellisen LW, Bird J, West DC, Soreng AL, Reynolds TG (1991) TAN1, the human homolog of the Drosophila Notch gene, is broken by chromosomal translocations in T-lymphoblastic neoplasms. Cell 66:649-661

Field LL, Tobias R, Thomson G, Plon S (1996) Susceptibility to insulindependent diabetes mellitus maps to a locus (IDDM11) on human chromosome 14q24.3-q31. Genomics 33:1-8

Frohman MA, Dush MK, Martin GR (1988) Rapid production of fulllength cDNAs from rare transcripts: amplification using a single gene-specific oligonucleotide primer. Proc Natl Acad Sci USA 85:8998-9002

Grant B, Greenwald I (1996) The Caenorhabditis elegans sel-1 gene, a negative regulator of $l i n-12$ and $g l p-1$, encodes a predicted extracellular protein. Genetics 143:237-247

Greenwald I, Rubin GM (1992) Making a difference: the role of cellcell interactions in establishing separate identities for equivalent cells. Cell 68:271-281

Greenwald IS, Sternberg PW, Horvitz HR (1983) The lin-12 locus specifies cell fates in Caenorhabditis elegans. Cell 34:435-444

Horton P, Nakai K (1996) A probabilistic classification system for predicting the cellular localization sites of proteins. Intell Syst Mol Biol 4:109-115

Jhappan C, Gallahan D, Stahle C, Chu E, Smith G (1992) Expression of an activated Notch-related int-3 transgene interferes with cell differentiation and induces neoplastic transformation in mammary and salivary glands. Genes Dev 6:345-355

Liang P, Pardee AB (1992) Differential display of eukaryotic messenger RNA by means of the polymerase chain reaction. Science 257:967-971

Liotta LA, Steeg PS, Stetler-Stevenson WG (1991) Cancer metastasis and angiogenesis: an imbalance of positive and negative regulation. Cell 64:327-336 chicken gelatinase (GEL), rabbit metalloproteinase-9 (MP-9), and rabbit matrix metalloproteinase-2 (MMP-2). Conserved amino acids are highlighted in black

Martin GR, Timpl R, Kuhn K (1988) Basement membrane proteins: molecular structure and function. Adv Protein Chem 39:1-50

Ozaki K, Kuroki T, Hayashi S, Nakamura Y (1996) Isolation of three testis-specific genes (TSA303, TSA806, TSA903) by a differential mRNA display method. Genomics 36:316-319

Ozaki K, Nagata M, Suzuki M, Fujiwara T, Miyoshi Y, Ishikawa O, Ohigashi H, Imaoka S, Takahashi E, Nakamura Y (1998) Isolation and characterization of a novel human pancreas-specific gene, pancpin, that is down-regulated in pancreatic cancer cells. Genes Chromosomes Cancer 22:179-185

Poston GJ, Gillespie J, Guillou PJ (1991) Biology of pancreatic cancer. Gut 32:800-812

Reponen P, Sahlberg C, Huhtala P, Hurskainen T, Thesleff I, Tryggvason K (1992) Molecular cloning of murine 72-kDa type IV collagenase and its expression during mouse development. J Biol Chem 267:7856-7862

Robbins J, Blondel BJ, Gallahan D, Gallahan R (1992) Mouse mammary tumor gene int-3: a member of the Notch gene family transforms mammary epithelial cells. J Virol 66:2594-2599

Tezuka K, Nemoto K, Tezuka Y, Sato T, Ikeda Y, Kobori M, Kawashima H, Eguchi H, Hakeda Y, Kumegawa M (1994) Identification of matrix metalloproteinase- 9 in rabbit osteoclasts. J Biol Chem 269:15006-15009

von Heijne G (1986) A new method for predicting signal sequence cleavage sites. Nucleic Acids Res 14:4683-4690

Walter MA, Spillett DJ, Thomas P, Weissenbach J, Goodfellow PN (1994) A method for constructing radiation hybrid maps of whole genomes. Nat Genet 7:22-28

Watanabe TK, Fujiwara T, Shimizu F, Okuno S, Suzuki M, Takahashi E, Nakamura Y, Hirai Y (1996) Cloning, expression, and mapping of TCTEL1, a putative human homologue of murine Tcte1, to $6 \mathrm{q}$. Cytogenet Cell Genet 73:153-156 